\title{
Speed Limits: The Architectural Design Possibilities of the 3D Printed Corner
}

\author{
ZACH COHEN \\ Massachusetts Institute of Technology, \\ Wentworth Institute of Technology
}

\begin{abstract}
This paper uses the example of the 3D printed corner to discuss an architectural design approach to the technical and material limits of 3D printing. The discussion begins by introducing the design of corners as a paradigm of the constantly evolving tension between architecture and materiality. The technical and material limits that define the 3D printed corner are presented along with a critique of current efforts to overcome these limits. The 3D printed corner is then situated within the history of architectural detailing. Finally, 3D printing experiments, at both desktop- and fullscale, are used to speculate on three alternative approaches to the 3D printed corner. The instrumental and architectural knowledge that underlies each approach is described along with the implications of each approach for the materiality of 3D printed designs.
\end{abstract}

\section{DEFINING THE LIMITS:}

\section{THE MATERIALITY OF THE CORNER}

In the development of architectural designs, the corner is often an occasion in which formal and tectonic logics meet the limitations of material systems-the design, for a moment, is confronted with its own materiality; one becomes inseparable from the other. In this way, the corner represents a paradigmatic architectural design challenge: How do we, architectural designers, carry our design concepts through the convergence of design and material constraints? Or to use the words of a famously meticulous contemporary architect: How do we design architecture in which "every touch, every join, every joint is there in order to reference the idea of the quiet presence of the work?" ${ }^{1}$ Digital fabrication has distanced architectural designers from hands-on craft; we find ourselves alienated from materials. Thus, in our current moment, the challenge to render every joint alive with its own materiality is particularly acute. How do we generate, materialize, and enhance design concepts within the constraints of digital fabrication machines?

3D printing would seem to be a fabrication method capable of sharpening the translation of architectural design ideas into material parts: "every touch, every join, every joint" is made by the same machine directly from our digital models. And yet, the discrepancy between 3D modelled corners and 3D printed corners demonstrates that the tension between architecture and materiality not only persists but has been complicated by the limitations of the machine. In this paper, I draw on historical precedents in architectural detailing and 3D printed corner experiments to show how an architectural design approach to 3D printing can reinvent its technical limits as design possibilities. In other words, I want to show that we can and should use the details of the machine to detail our designs.

\section{THE “PROBLEM" OF THE 3D PRINTED CORNER²}

Why are digitally fabricated corners not precise reproductions of digitally modelled corners? ${ }^{3}$ In desktop 3D printing, the imprecision is caused by an (almost) imperceptible delay. This delay occurs because the extruder cannot change directions instantaneously. Such a change in acceleration would exert a destructively high amount of torque on the machine parts. In other words, the speed of the 3D printer is limited by the machine's materiality. Instead, when the extruder approaches a corner, it must slow down or pause in order to smoothly assume its new trajectory. The pause expresses the invisible joint that ties the two sides together. Since locomotion is delayed at the corner but extrusion is not, there is a small material build-up, or "swell," that occurs (figure 1a). Alternatively, if the machine is not slowed down enough, the nozzle will stagger around the corner, causing small ripples, or "ringing," in the otherwise evenly printed surface. ${ }^{5}$ The speed of the printing process-whether slow or fast-has material consequences.

At the scale of desktop 3D printing, such small material imperfections may seem inconsequential. However, as architecture students learn in their freehand drawing exercises, the ways in which lines meet influence the reading of our representations: the definition of corners is tied to the definition of the design. Further, when 3D printers are scaled from desktop- to full-scale, the material consequences of the printing speed are amplified. In concrete 3D printing, for example, the machine also needs to account for the gravity of the material: as the machine accelerates around the corner, the material accelerates downwards. The machine has to wait for the material to land. If the machine corners too quickly, the concrete will twist or tear (figure 1b). Here, transgression of the machine's speed limit can actually dematerialize the designs that we 3D print. 

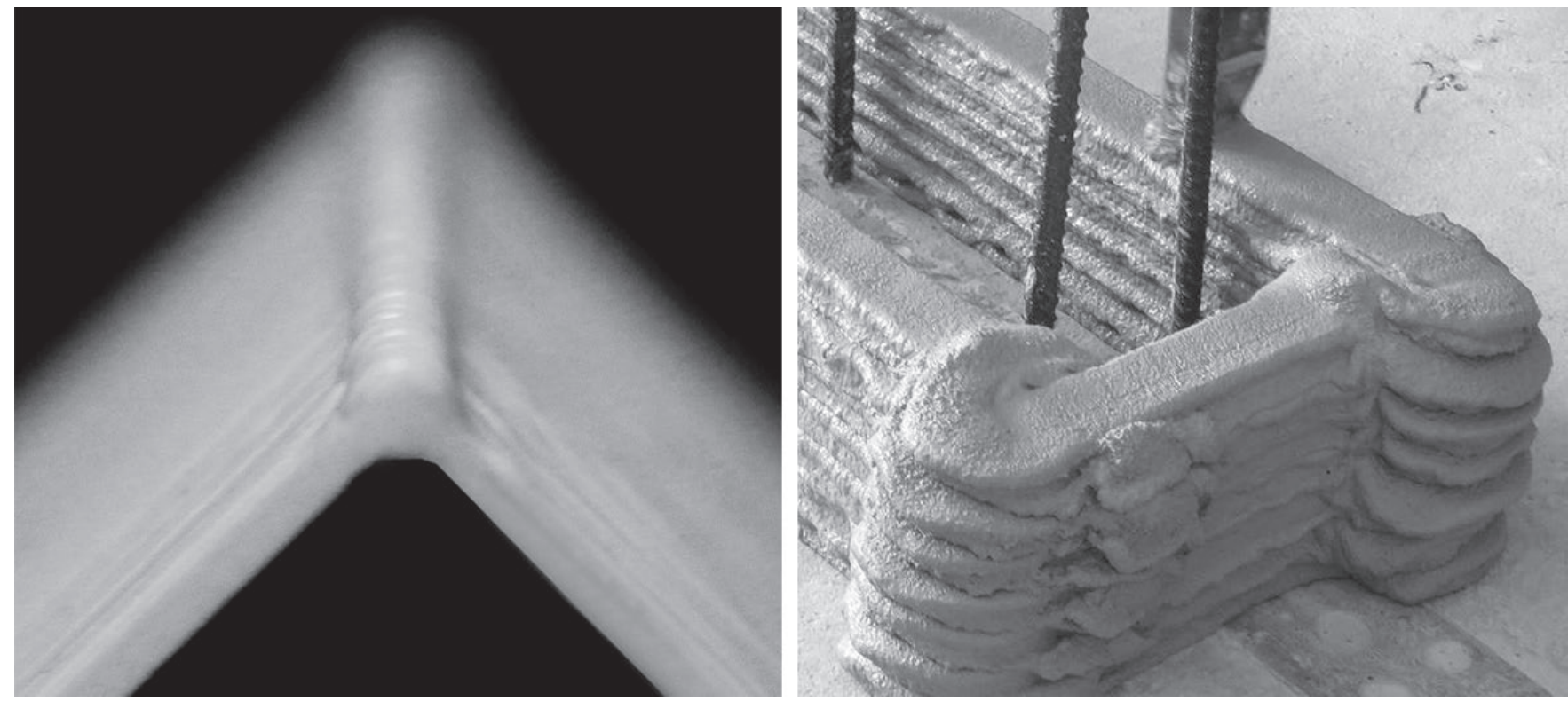

Figure 1. Left, Small delays at directional changes cause the 3D printed thing to "swell" at the corners; right, The corners of 3D printed concrete twist and/ or tear when they are printed too quickly. ${ }^{5}$

In the current practice and research of 3D printing, the swells, ripples, twists, and tears unintentionally alter the materiality of our 3D printed designs. I intend to illustrate that we can transform these technical glitches into architectural details if we understand and work with the constraints that cause them to materialize. Further, I hope to demonstrate that such fundamental understanding can reveal opportunities to unite 3D printing workflows with design methods that are at the foundation of architectural knowledge.

Some designers and technologists aim to solve the 3D printed corner "problem" by optimizing the deceleration of the extruder in order for it to turn the corner seamlessly. ${ }^{6}$ They eschew architectural detailing for digital computation; they try to stop-rather than stop in-the delay. We need to understand that the digital fabrication machine can only approximate digital precision; the delays, along with their material consequences, can never fully be removed. Further, we need to remember that a 3D print is a material thing and not a digital thing incarnate. Material things take time to materialize. If we consistently try to make our 3D prints materialize as quickly and precisely as our mouse-click modeling, we will lose the understanding of materials that is crucial to the development and realization of architectural designs-the 3D printer will only ever be a microwave for "pre-cooked" models. Alternatively, if we embrace the delays inherent to 3D printing as necessary and productive constraints, we open the possibility of manipulating them to uncover materiality that better represents our design thinking. The 3D printer can become an instrument for architectural design.

\section{CORNERING THE DESIGN CONCEPT}

The history of architectural detailing shows us that the convergence of design and material constraints has been celebrated, rather than bemoaned or feared, through various forms of ornamentation and/or craft that reinforce design concepts. For example, when ancient Classical Greek builders introduced stone into Doric temples in the fifth century, the main beams now had to extend past the centerlines of corner columns in order to support the heavier material. ${ }^{7}$ Subsequently, the ornamental reliefs at the corners of the beams shifted out of their regular spacing and out of alignment with the columns below. The symmetry about the corner, which was central to the desired visual perception of the temple, was broken. Greek architects deployed two strategies to restore the symmetry: widen the outermost reliefs until the spacing between reliefs was even again, or shift the corner column and the relief above inwards until they realigned and, as a result, lose the repetitive interval between columns. ${ }^{8}$ Both corner strategies were designed to absorb the impact of material consequences with minimal distortion of the ordering principles. In turn, the corners became the exceptions that proved conceptual rules.

A historical example that is closer in time and spirit to the current moment in digital fabrication can be found in the work of the twentieth-century German-American architect Mies van der Rohe. Following the Industrial Revolution, new material technologies introduced formal possibilities: corners could now be extruded, precast, and, even, removed. Instead of Classical orders, material became both the expression and the limit of architectural design. Materiality became a concept unto itself. ${ }^{9}$ Mies van der Rohe's work inherited the advancements of the Industrial Revolution in order to revitalize conceptual 


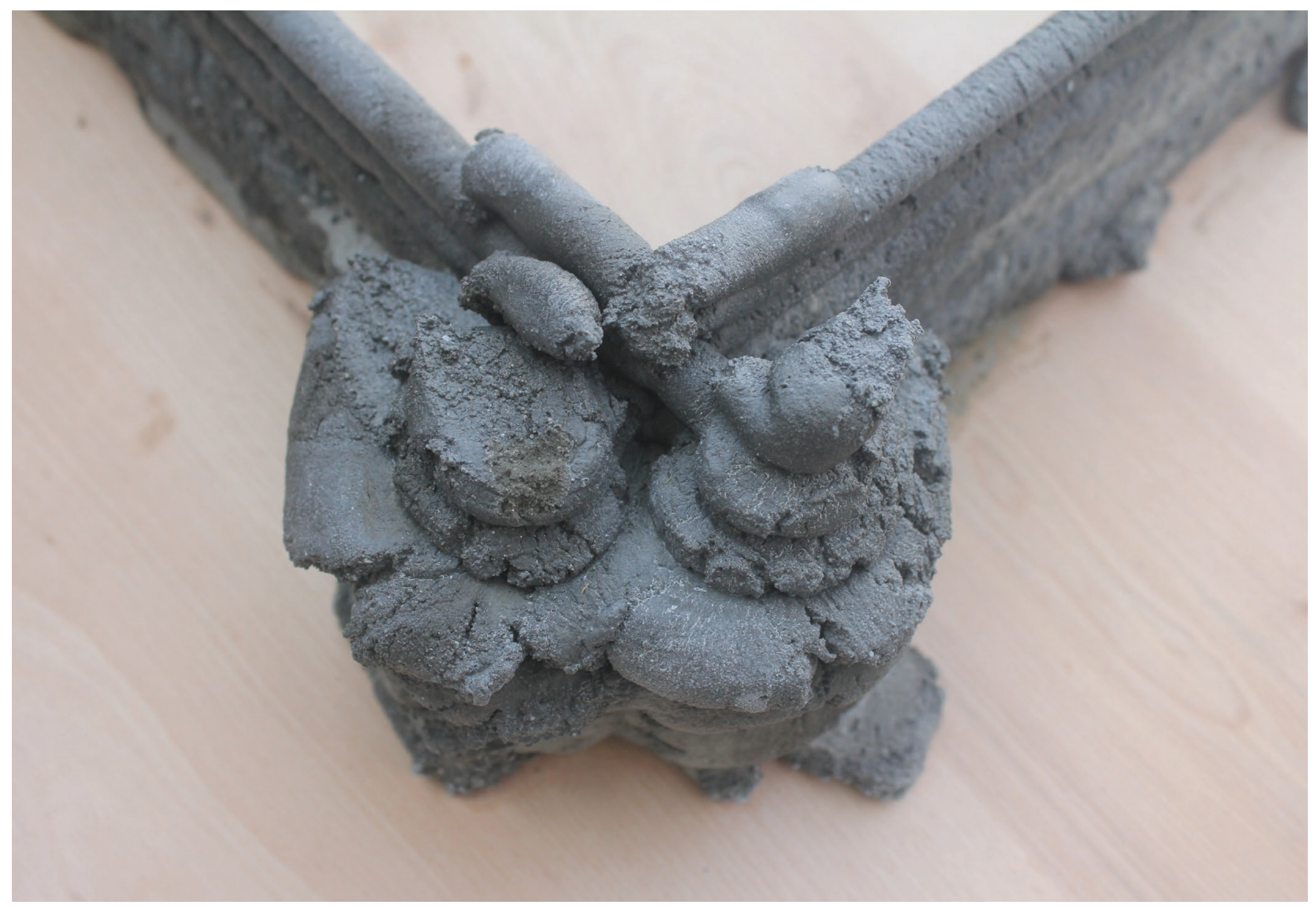

Figure 2. 3D printed concrete lines are extended past one another in order to create an ornament at the corner.

thinking in architecture. In the buildings on the campus of the Illinois Institute of Technology (IIT), for example, Mies aimed to "express" the load-bearing steel structure that enabled the clear span interior spaces; however, the steel had to be fireproofed and therefore could not be exposed. As a result, steel panels were used to cover the fireproofing and hint at the material within. At the corners, the steel panel folded back to create a "negative corner," or void, that denoted where the columns are. ${ }^{10}$ Mies would go on to employ similar details in his steel skyscrapers-for example, in One Charles Center in Baltimore, in which he removed the corners in order to emphasize the thinness of the curtain wall. ${ }^{11}$ In Mies' architectural portfolio, the absence of material at the corner presents the materiality-and the concept-of the building.

The examples of the Greek temples' columns and Mies' steel channel details demonstrate that the architectural design approach to making necessitates that we work within material constraints - not against them-and that we regularly learn what those constraints are. We need to see the 3D printed corner through the lens of this approach and begin to work with the speed limits (so to speak) of the material and the machine at hand. Such an approach will enable us to speculate on new corner details that embody and define our designs.

\section{INSTRUMENTALIZING LIMITS}

I would like to offer three alternative approaches to 3D printing corners that I found while experimenting with design workflows that integrate architectural and instrumental knowledge. ${ }^{12}$ In the context of my own experiments, instrumental knowledge entails an understanding of the reasons for the "corner delay" (which I discuss above) and a sense for the ways in which an architect can work beyond (or prior to) the machine's default settings. In these experiments, I combine this knowledge with architectural design concepts in order to innovate the materialization of the 3D printed corner.

\section{LEARNING FROM THE PRINTED LINE}

The first year of architecture school is filled with exercises that teach concepts and techniques of two-dimensional representation: draw a human figure without picking your hand up from the page; cut a section through a found object; construct a two-point perspective. As mentioned above, one thing that students will learn from these exercises is that the ways in which they draw corners affect the dimensionality of their representations. At first, students might inadvertently round their corners or draw lines that don't quite meet; to ensure that corners are not left unresolved, professors might instruct a student to extend 

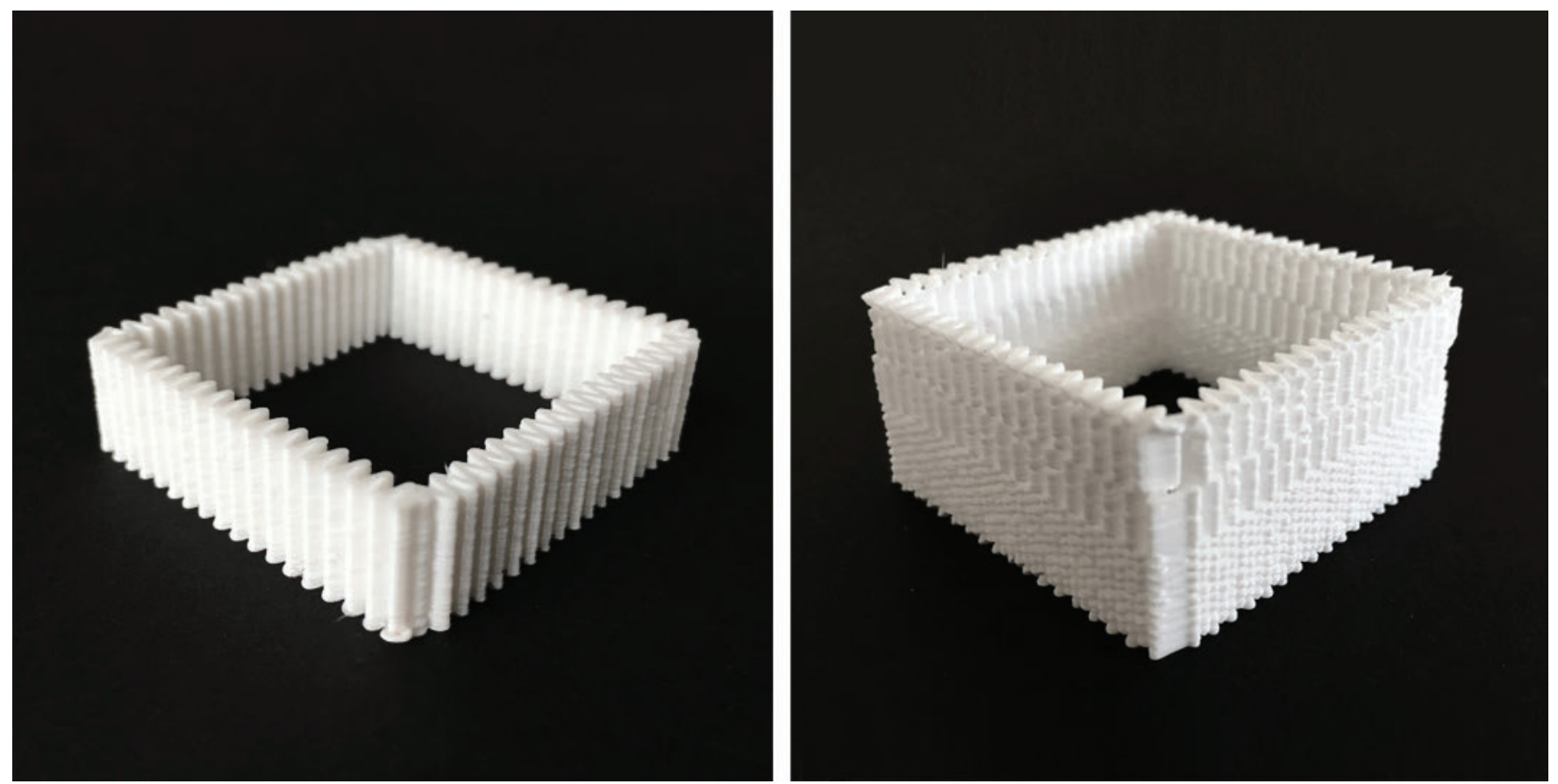

Figure 3. In these 3D printed plastic tests, the extruder was instructed to print squiggles instead of straight lines. The squiggles absorb the inevitable imperfections of the 3D printed corner

lines slightly past one another. If we reject the notion that all 3D printed lines must be printed continuously and sequentially, i.e., that we cannot print open line segments (as proprietary software leads us to believe), then we can apply this drawing technique to 3D printed corners. For example, we can reorder lines to start and end at different points, and therefore free them to overlap-rather than meet-at their intersections (figure 2). These overlaps can ornament our corners with interlocking swells or provide additional structure for subsequent layers. This rethinking also points to the possibility of the 3D printer as an educational tool that can assist in teaching relationships between two- and three-dimensional representation.

We can and should draw another lesson from freehand drawing: the benefits of the squiggly line. Squiggly strokes overcome the impossibility of drawing perfectly straight lines by embracing inevitable imperfections (figure 3 ). The small deviations from the direct path also imply invisible margins that enable us to create a line that is often straighter overall. Here, we can recall the ways in which the architects of Doric temples made small shifts at corner assemblies in order to preserve the building's global geometry. The extruder of a 3D printer can also be made, or programmed, to waver slightly during its extrusion. Such oscillation would absorb unforeseen blemishes, e.g., the aforementioned ringing, and would texture the otherwise monolithic surfaces of our 3D printed things. In other words, we can make several temporary, intermittent directional changes that simultaneously build up to and take away from the material consequences of the more permanent directional change at the corner.

\section{LEARNING FROM MANUAL DEPOSITION}

If we contextualize the layers of conventional 3D printing within other component-based systems that are already embedded within the material palette of architectural design (e.g., bricks or stones), we will see that pauses are inherent to the 3D printing process. A component-based system cannot be piled all at once. Instead components must be stacked, or, manually deposited: one component is placed, then another, and another, and so on-there are as many pauses as there are components.

Before a designer can 3D print she must pass her digital model through software, which "slices" the model into a series of two-dimensional contours, subdivides each of those contours into a list of three-dimensional points, and then attaches instructions for how the model should be printed. The machine prints stacks of points. Yet the quickness and continuity of the deposition ensures that we only ever perceive lines(i.e., the layers) - that is, until we turn the corner, and the point reveals itself as a small bump of material. What if, then, instead of instructing the machine to interpolate a curve between these points, we instructed it to stop at every point-what if we let the material do the interpolation? What if there were only bumps, only pauses? The bump would no longer be an anomaly but the component itself (figure 4). Further, the convergence of bumps, i.e., the corner, could produce unexpected aesthetics and irregular joints.

\section{LEARNING FROM THE VOID}

Current 3D printing software convinces us that digital models have to be "watertight," or without holes, in order to be printed. However, as we learned earlier from the precedent 


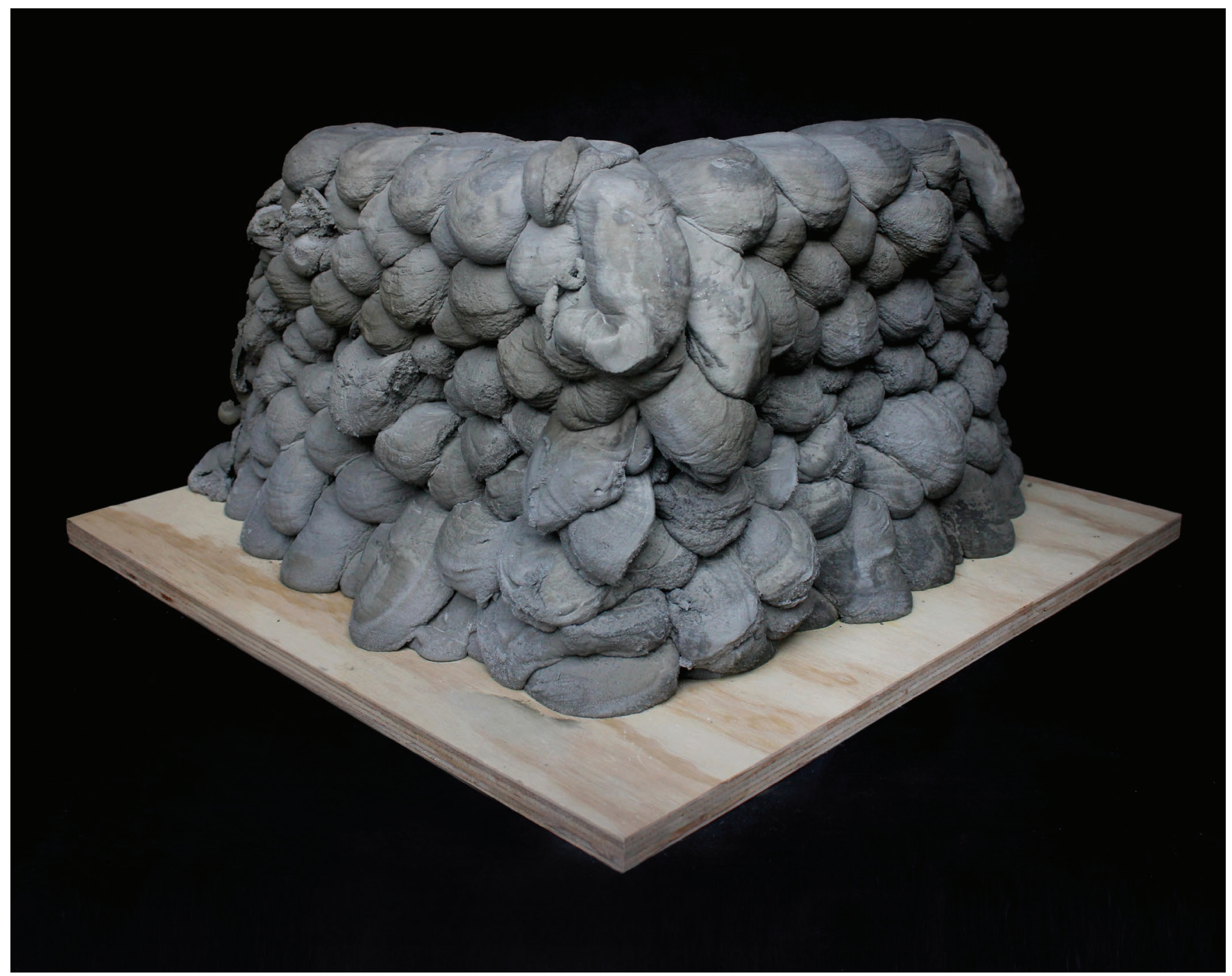

Figure 4. The "swell" becomes the architectural component in this half-scale experiment in 3D printed concrete.

of Mies' IIT buildings, the absence of material can suggest materiality. We can infer from IIT that the Miesian approach to the 3D printed corner would be to remove the corner entirely. In other words, if we can't 3D print the corner that we envision, then we shouldn't 3D print a corner at all. We could interpret such an approach as the elimination of all sharp edges from our 3D printed designs. ${ }^{13}$ However, such a uniform resolution does nothing to enhance the nuances of our designs; even worse, it could smooth their pointedness. I am more interested in the actual removal of entire parts of 3D printed corners; not by extending or multiplying the corner delay (as was discussed in the "interlocking swells" or "bumpy pauses"), but by delaying the materiality of the corner indefinitely. The removals could be incorporated in order to deliberately draw attention to other parts of the 3D printed design, similar to the ways in which voids were deployed in Mies' skyscrapers. More importantly, such subtraction within an additively manufactured thing could introduce a porousness that softens the boundaries of the 3D print and the 3D printing process (figure 5).

\section{MOVING FORWARD}

The architectural design approach to 3D printing corners, and 3D printing in general, that I have advocated in this paper is defined by working with the speed limits of the machine in order to produce materiality that enriches design concepts. The development of such an approach requires that both students and practitioners of architecture reflect on the historical, material, and technical knowledge that is fundamental to our work and, further, that we intertwine this knowledge with deeper insights into the inner workings of the machine. At the same time, we need to resist the compulsion to repeatedly close the irrevocable rift between digital and material things, and to begin to collectively remember 

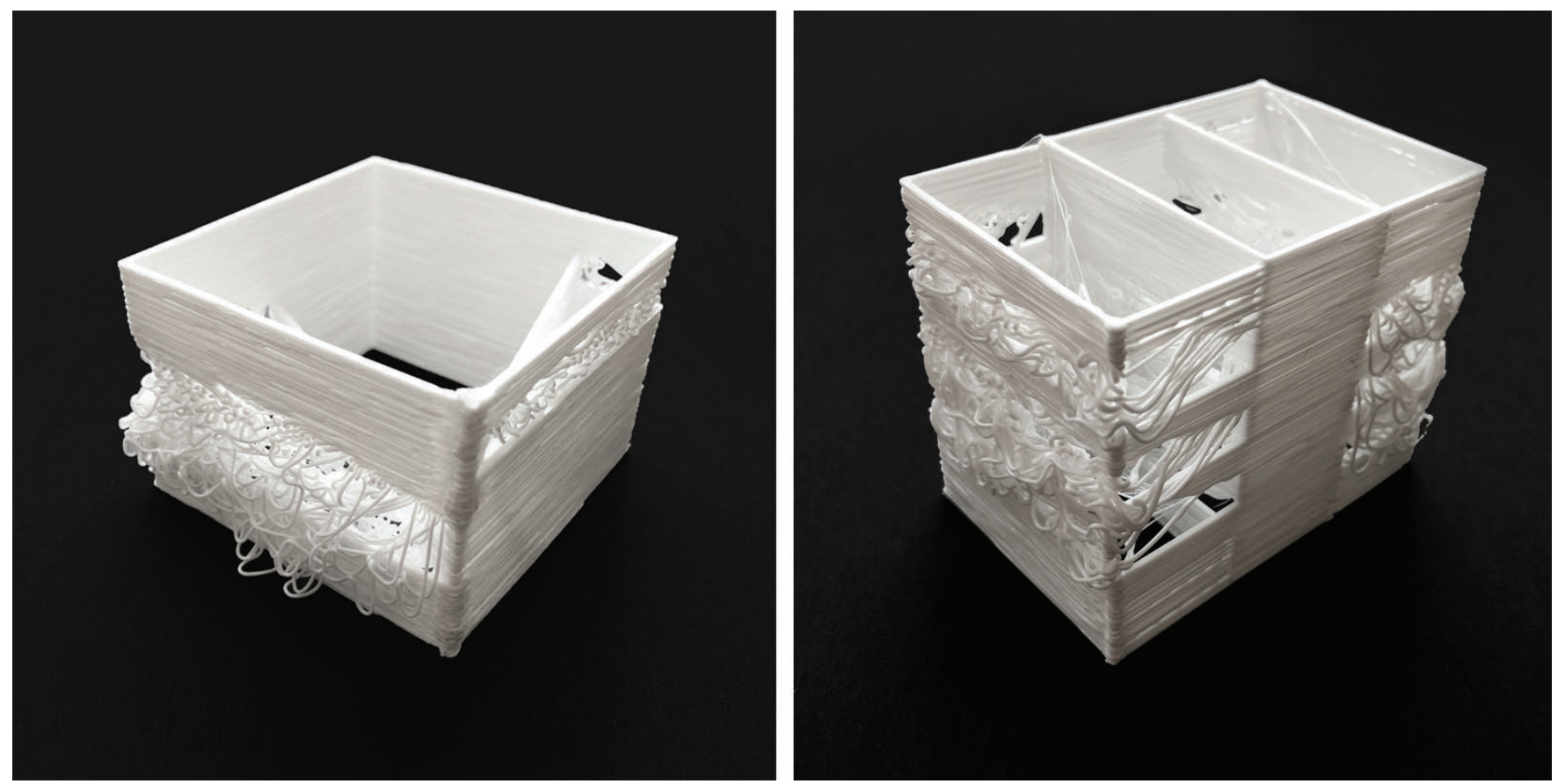

Figure 5. The corners are selectively removed in order to introduce softness into the rigidity of the 3D printed plastic thing.

that materialization takes time. Both as a prototypical architectural design challenge and as a moment of necessary deceleration, the corner is not a problem to be solved. On the contrary, I have argued, the corner is the ideal opportunity for architects to slow down, reflect, and redirect our interactions with 3D printers, so as not to further distance ourselves from the matter of architectural design. Moving forward, we should turn around and ask: How can the 3D printed corner become the moment in which we rethink the relationship between architecture and materiality? How can the corner become what it is?

\section{ENDNOTES}

1 Peter Zumthor, Thinking Architecture (Basel, Switzerland: Birkhauser, 1998), 14.

2 The Oxford English Dictionary defines problem as "a matter or situation regarded as unwelcome, harmful, or wrong and needing to be overcome; a difficulty." See “Problem, n.3a," entry, OED Online, accessed August 28, 2019. www.oed.com/ viewdictionaryentry/Entry/11125.

3 The imprecision is not exclusive to 3D printers. Subtractive digital fabrication machines, such as routers or mills, will always produce slightly rounded interior corners because of the circular cross section of the end mill. These corners often have to be sharpened by hand.

4 Daniel Halvorson, "A Solid Foundation for: High-Quality Corners," Printed Solid 27 (November 2016). www.printedsolid.com/blogs/ news/a-solid-foundation-for-high-quality-corners.

5 There is a third possible cause of distorted 3D printed corners, which is warping. However, for the sake of concision, this imperfection is not discussed.

6 Rik Hammer, "Test with 3D-Printed Concrete Formwork a Success," Heijmans 16 (June 2016). https://www.heijmans.nl/en/news/ test-3d-printed-cement-formwork-success/.

7 Zheeshan Y. Ahmed, et. al, "Design Considerations Due to Scale Effects in 3D Concrete Printing," paper presented at the 8th ASCAAD Conference, London, UK, November 7-8, 2016.

8 A.W. Lawrence, Greek Architecture: Fifth Edition, revised by R.A. Tomlinson (New Haven, CT: Yale University Press, 1996), 72.
9 Peter Eisenman, “There Are No Corners After Derrida," Log 15 (2009): 114.

10 Gail Peter Borden, “Material Precedent: The Typology of Modern Tectonics," paper presented at the 97 th ACSA Annual Meeting, Portland, OR, March 26-29, 2009

11 Carsten Krohn, Mies van der Rohe: The Built Work (Basel: Birkhauser, 2014), 115.

12 Krohn, 185

13 The architect and mathematician Andrew Witt defines instrumental knowledge in terms of "a more narrow understanding of the procedures to successfully operate a certain type of technology, which would include the ability to operate a software, program, script, process, tool, instrument, or machine to intended effect." See: Andrew Witt, "A Machine Epistemology in Architecture: Encapsulated Knowledge and the Instrumentation of Design," Candide 3 (2010): 37-87.

14 Some researchers have already adopted this approach, see: A.S.J. Suiker, "The Mechanical Performance of Wall Structures in 3D Printing Processes: Theory, Design, Tools, and Experiments," International Journal of Mechanical Sciences, 137 (2018): 145-170. 